\title{
A CASE OF TENSION PNEUMOTHORAX COMPLICATING STAPHYLOCOCCAL PNEUMONIA AND EMPYEMA THORACIS
}

\author{
BY \\ R. SPENCER \\ From Alder Hey Children's Hospital, Liverpool
}

(RECEIVED FOR PUBlicAtion AUGUST 8, 1952)

Empyema thoracis is seldom a primary condition. It must necessarily be considered against the background of infection of the respiratory tract. Staphylococcal pneumonia along with its major complication, empyema, has been recognized as a separate clinical entity for some years, although Agnes Macgregor as recently as 1936 described 10 cases in support of this conception. The extensive use of sulphonamides in the treatment of respiratory infections succeeded in throwing into relief cases unresponsive to these drugs. Not only has there been a relative increase in the incidence of staphylococcal pneumonia but several authors, including Guthrie and Montgomery (1947), have shown that the increase is also a real one.

Secondary staphylococcal pneumonia is distinguished from the primary type by being a complication of a staphylococcal focus of infection elsewhere in the body, for example, osteomyelitis. For the aetiology, clinical features, mortality, etc., in relation to treatment reference may be made to papers by Gibson and Belcher (1951), Guthrie and Montgomery (1947), Kanof, Kramer and Carnes (1939), Dickie (1938) and Chickering and Park (1919).

Two features of staphylococcal pneumonia stand out clearly. First, all accounts of the disease stress the frequency of empyema. Kanof et al. (1939) look upon empyema as an integral part of the disease rather than as a complication. In their review of 15 primary and 12 secondary cases $87 \%$ of the primary and $58 \%$ of the secondary types developed empyemata. Secondly, a strong predilection for the youngest age group is shown, especially by the primary pneumonia. Kanof et al. (1939) discussed 23 cases of primary and 12 cases of secondary staphylococcal pneumonia. In the primary cases $70 \%$ were under 1 year old, $9 \%$ were over 5 years. In the secondary cases, $41 \%$ were under 1 year and
$25 \%$ were over 5 years. The patients were older in the series of secondary pneumonias.

The high incidence of empyema may be related to the extreme youth of the patients. Cohen (1938) suggested that the pleura easily became infected since the layer of lung tissue was very thin. It is generally considered, however, that in many cases the empyema is caused by the rupture of an abscess in the lung into the pleural space. Macgregor (1936) supported this view from the post-mortem findings in 10 fatal cases. She pointed out that the inflammatory process in the lung may be confined to a small area while the whole pleura is often full of pus. Blumenthal and Neuhof (1946) described a 3-year-old girl who had a tension pneumothorax. Stab thoracotomy was performed to relieve respiratory and circulatory distress. Next day there was a sudden expulsion of a muco-purulent plug from the drainage tube, followed by a gush of air lasting some minutes. The authors considered that this event indicated the sudden rupture of another abscess into the pleura. They found in another patient, a 6-year-old boy, a narrow extension of an empyema leading directly to a small collection of pus within necrotic lung.

Since abscesses can discharge spontaneously into the bronchi, it is reasonable to expect that they can burst into the pleural space. The frequent incidence of pyopneumothorax favours this conception. Since, however, localized empyemata also occur not all cases take place in this way. Protective adhesions may have time to form and invasion of the pleura be more gradual. The virulence of the organism, the resistance of the patient, and the efficacy of treatment must influence the incidence and size of an empyema.

The presence of air in the pleural space following or during the course of staphylococcal pneumonia 
means the occurrence at some stage of a bronchopleural fistula. It is only possible, in reviewing the literature, to arrive at an approximate idea of the frequency of empyema and pyopneumothorax complicating pneumonia. The main reason for this is the varying virulence of the organism, different methods of treatment (especially of empyema) and the evolution of new chemotherapeutic drugs. Some of the bigger series of cases are analysed here with special reference to pyopneumothorax.

Macgregor (1936) in her series of six nonfulminating but fatal cases out of a total of 10 patients with primary staphylococcal pneumonia reported empyema occurring in $100 \%$. Air was also present in three, and in each of these a bronchopleural fistula was identified at necropsy.

In the description of 23 cases of primary staphylococcal pneumonia and 12 of secondary pneumonias, Kanof et al. (1939) had only two cases of pneumothorax occurring before chest aspiration had been attempted; one in a baby girl of 7 months who died, and the other in a girl of $\mathbf{3 0}$ months who recovered. No case of tension pneumothorax occurred.

Hochberg and Kramer (1939) found one case of bronchopleural fistula out of 33 cases of staphylococcal empyema. The mortality in the whole series was $22 \%$. Their conclusions were that mortality was proportional to the inadequacy of drainage of the empyema. They were not of the opinion that rupture of pulmonary abscess was the direct cause of an empyema. Under 2 years of age complications associated with empyema were more frequent than above that age.

Clemens and Weens (1942) described six cases of primary staphylococcal pneumonia. Every patient acquired an empyema. Four had pyopneumothorax. All died from toxaemia.

Riley (1944), reviewing 29 cases of empyema caused by staphylococci, made the observation that $76 \%$ occurred in the first year and $40 \%$ in the first six months. Of 22 infants under 1 year of age, eight died. Of seven over 1 year, two died. No mention is made of pneumothorax or pyopneumothorax in his review.

Ladd and Swan (1943) made an important contribution to the subject in their study of 33 cases of empyema. Twenty-one were under 1 year and 12 were under 4 months. The most important factor influencing the mortality seemed to be the age of the patient. Of 12 patients less than 4 months old, eight died $(67 \%)$. Of 21 patients over 4 months old, only one died $(5 \%)$. One case described was that of a $2 \frac{1}{2}$-year-old boy who recovered after fulminating laryngo-tracheobronchitis requiring tracheotomy and bronchoscopy. He had bilateral empyema and bilateral tension pneumothorax in addition to underlying pulmonary abscesses. The treatment of their cases was chiefly by drainage and sulphonamides.

Blumenthal and Neuhof (1946) carefully analysed a series of 40 cases of primary staphylococcal pneumonia. Eight were fulminating cases allowing no time before death for the development of advanced pulmonary or pleural changes. A second group of 11 were less overwhelming in their course. Each patient had an empyema in which the whole of one pleural space was affected. The range in age was from 1 month to 3 years. Seven patients died. Five had pus alone in the pleura: three of these died. Six had pyopneumothorax from which four died. In two of these there was tension and a mediastinal shift. Eight of the total of 11 were described as having a bronchopleural fistula.

A third group of 17 were cases in which encapsulated abscesses or empyemata had developed. Fourteen of these were cases with empyema. Their range of age was 1 month to $12 \frac{1}{2}$ years, the average age being almost twice that of the previous group. Twelve had pus only, while two had pyopneumothorax. No case of tension pneumothorax was described. Altogether 13 out of the 17 cases had bronchopleural fistula verified by operation for drainage or by radiography using lipiodol instilled via the empyema track. There was no mortality in this group.

Lastly a fourth group was described of four chronic and subacute cases. Each of the four had an empyema. The ages were 7, 7,2 and 6 years respectively. Three of the four had a bronchopleural fistula, but there was no case of pneumothorax in this small group.

Altogether the mortality in the 40 cases was 15 (or $35 \%$ ). Eight of the 15 did not acquire empyemata for reasons already stated. Four had pyopneumothorax and three simple empyema (not encapsulated). The numbers are too small for many conclusions to be drawn. They do, however, show that the most serious cases are those in which the whole pleura is flooded with pus, and that when this occurs bronchopleural fistula is probably present and there is a likelihood of air also escaping into the pleura. The presence of air is not in itself an indication that the chance of survival is less. It seems rather that the important feature is the total filling of the pleural space.

Davis, Hyman and Ruhstaller (1947) reported seven cases of primary staphylococcal pneumonia in patients whose ages ranged from 18 days to 11 years. All were complicated by pneumonia. No case of pyopneumothorax was described. One infant aged 2 months died. 
Gibson and Belcher (1951) stated that in their experience empyema occurs in $10 \%$ to $30 \%$ of adults suffering from primary staphylococcal pneumonia and $50 \%$ of children. In 10 cases of pneumonia there were four of empyema, one of which was a pyopneumothorax. No patient was under 14 years of age and all recovered. They considered pyopneumothorax to be relatively common in children and that it was a major factor causing death, prognosis being little affected by any form of treatment. Tension pneumothorax, they pointed out, is sometimes difficult to distinguish from a large pulmonary tension cyst, but they did not consider the differentiation of any great moment so far as treatment was concerned. They supported the 'check-valve' conception of air entering the pleura on inspiration but being unable to escape during expiration in the same manner that large cysts in the lung may develop.

The chief danger from tension pneumothorax is the circulatory embarrassment caused by mediastinal displacement. Blumenthal and Neuhof emphasized the point that the tension in the pleural space might rise with dramatic suddenness causing symptoms of shock, cyanosis and circulatory failure. Little has been written on the method of treating tension pneumothorax. Blumenthal and Neuhof advised prompt measures to relieve the tension and to restore the mediastinum to its normal position. At the same time it is equally important to avoid a sudden opening of the pleural space to atmospheric pressure since there is in these cases a bronchopleural fistula and re-expansion of the lung will obviously be prevented. The best method would appear to be a gradual withdrawal of the air and lowering of the pressure in order to avoid the risk of the mediastinum suddenly swinging over with its attendant danger of mediastinal flutter, and to encourage re-expansion of the lung. Some of these points are illustrated in the following case history.

\section{Case Report}

The patient J.J.N., a girl, was born on July 31, 1951. She was admitted to hospital at the age of 7 months, on February 25, 1952.

Delivery was normal after a normal pregnancy. Her weight at birth was $7 \mathrm{lb}$. $6 \mathrm{oz}$. She was breast fed for seven months and was partly weaned on admission to hospital.

Twenty-four hours after birth the patient showed evidence of haemolytic disease, melaena and haematemesis. Rh-positive group $\mathrm{O}$ blood, $200 \mathrm{ml}$., was given intravenously. The condition cleared satisfactorily and the haemoglobin was $100 \%$ on the seventh day.

Both parents and their four older children were well at the time of the patient's admission to hospital.

The social conditions were unsatisfactory. Two rooms only were available for the whole family, one used as living room and the other as bedroom. There were three beds for the use of them all.

Five days before admission the patient suffered from a running nose. She was noticed to have a sore mouth and would not suck. Breathing became laboured 48 hours before she was admitted into hospital with a diagnosis of pneumonia. 'Sulphatriad" had been administered during the five days of her illness.

On examination in hospital the child was pale and toxic, and the alae nasi were acting vigorously with inspiration. Respirations were grunting. There was impaired respiratory excursion of the right side of the chest. The apex beat of the heart was seen to be half an inch outside the nipple line in the fourth intercostal space. On percussion, there was stony dullness of the right chest in front and behind. On auscultation, breath sounds were tubular over the right side of the chest.

On abdominal examination, the spleen was just palpable. Apart from purulent rhinitis, there were no other abnormal physical signs. The temperature on admission was $99^{\circ} \mathrm{F}$, the respiration rate 60 and the pulse rate 140 . A diagnosis of pneumonia with pleural effusion was made.

Pathological Investigations. Pus from the pleura was examined on two occasions. On February 26 the specimen was yellow, and a direct film showed debris. numerous degenerate cells and large numbers of Grampositive cocci. On culture a profuse growth of Staphylococcus aureus (coagulase positive) was seen, and was penicillin- and streptomycin-sensitive.

The second specimen, also yellow, was seen on February 28. A direct film showed debris with large numbers of polymorph and mononucleated cells and numerous Gram-positive cocci. On culture there was a moderate growth of Staphylococcus aureus (coagulase positive). The organism was sensitive to penicillin, streptomycin, chloromycetin and aureomycin.

Blood cultured on March 6 showed no bacterial growth after eight days. On March 18 a full count gave $\mathrm{Hb} .78^{\circ}$ 。 $(11.5 \mathrm{~g} . \%$ ): leucocytes 12,000 per c.mm. (polymorphs, 6,960 or $58 \%$, eosinophils, 240 or $2 \%$, lymphocytes, 4,560 or $38 \%$, monocytes, 240 or $2 \%$ ).

Radiological Reports. On April 8 the pneumothorax was much smaller and was confined to the right upper zone, the right lung having re-expanded to fill the rest of the chest. The heart and mediastinum had returned to the mid-line and the herniation of the right pleura to the left side was not shown. A small amount of fluid remained at the right base (Fig. 5).

On May 7 the right lung was completely re-expanded with obliteration of the right costophrenic angle. There appeared to be some lipiodol residuum also. The eighth and ninth ribs had been resected surgically in the posterior axillary line (Fig. 6).

Treatment and Progress. On February 26 a paracentesis thoracis was performed and $75 \mathrm{ml}$. of thin, yellow, purulent fluid was aspirated, and proved the diagnosis of empyema. Penicillin, 750,000 units, was instilled into the pleural cavity. 


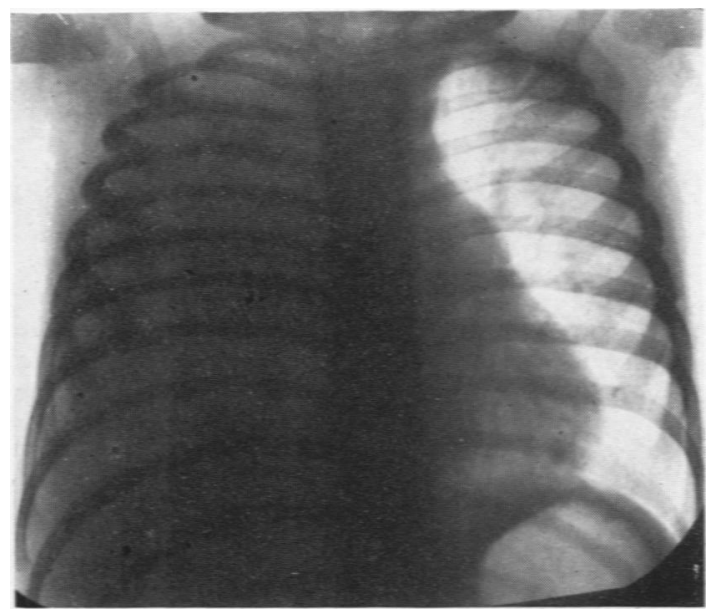

Fig. 1.-Radiograph demonstrating homogeneous opacity of the right side of the chest and the heart slightly displaced towards the left.

A radiograph (Fig. 1) of the chest showed a homogeneous opacity of the right chest, and the heart appeared to be slightly displaced to the left.

The patient was put on a course of penicillin starting on February $25,500,000$ units daily was given in divided doses six-hourly. This was continued until March 6. Streptomycin, $20 \mathrm{mg}$. per lb. body weight (estimated at 16 lb.) was also given daily from February 28 to March 6. Terramycin was given orally, $200 \mathrm{mg}$. daily from February 28 to March 6.

The pathological report of the pus taken on February 26 was that on culture there was a profuse growth of

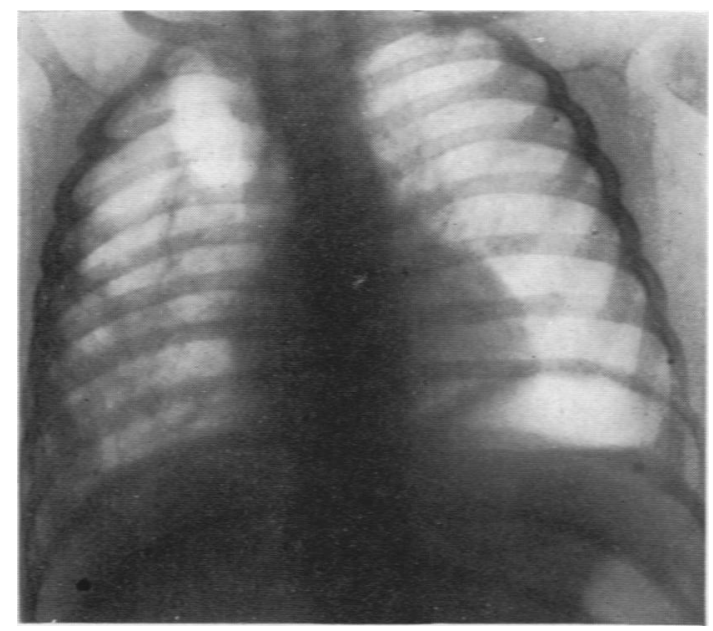

FKG. 2-Radiograph during intercostal drainage and before instillation of lipiodol. There is a translucent area in the right upper chest.
Staphylococcus aureus, coagulase-positive, penicillin- and streptomycin-sensitive.

Chest aspirations were performed daily. On February $27,80 \mathrm{ml}$. thin pus was aspirated: on February $28,58 \mathrm{ml}$. was aspirated and 125,000 units penicillin instilled: on February $29,10 \mathrm{ml}$. of pus was aspirated, and 125,000 units of penicillin instilled.

The patient's clinical condition was improving. The temperature, which had ranged from $99^{\circ} \mathrm{F}$. to $101^{\circ} \mathrm{F}$., began to settle and fluctuations were less marked. Pus examined bacteriologically on February 28 gave the same organism which was sensitive to penicillin, streptomycin. chloromycetin and aureomycin.

On March 1 paracentesis of the chest failed to obtain any pus and it was thought that it was too thick to flow through the needle. A radiograph showed a collection

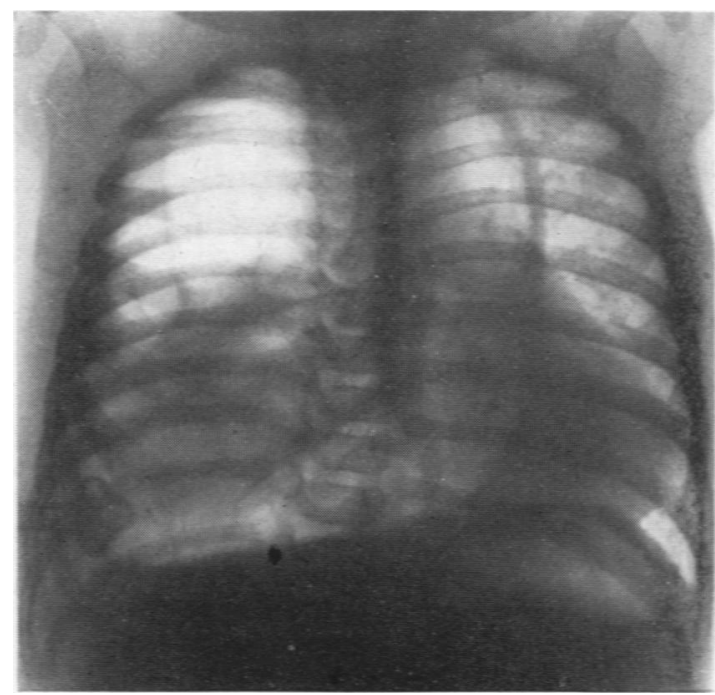

Fig. 3.-Radiograph during intercostal drainage showing a translucent area of the right upper chest extending over to the left side. and mediastinal displacement.

of fluid as high as the fourth rib: there was a fluid level and air above it. Using $1 \%$ 'novocaine' as local anaesthetic, a vertical incision was made over the seventh or eighth intercostal space in the posterior axillary line and intercostal drainage was established by means of a rubber catheter (No. 6 Jacques). The skin was closed around the catheter and underwater drainage into a bottle of antiseptic solution at the bedside was employed. On each subsequent day 125,000 units of penicillin were instilled into the pleura via the intercostal drain until March 7. The empyema cavity ceased to drain and a de Pezzer type of catheter was inserted in place of the Jacques catheter.

Figs. 2 and 3 are radiographs taken during the period of intercostal drainage.

In spite of the de Pezzer catheter, drainage continued to be unsatisfactory, and on March 18 about $5 \mathrm{ml}$. of lipiodol was run through the drainage tube into the 


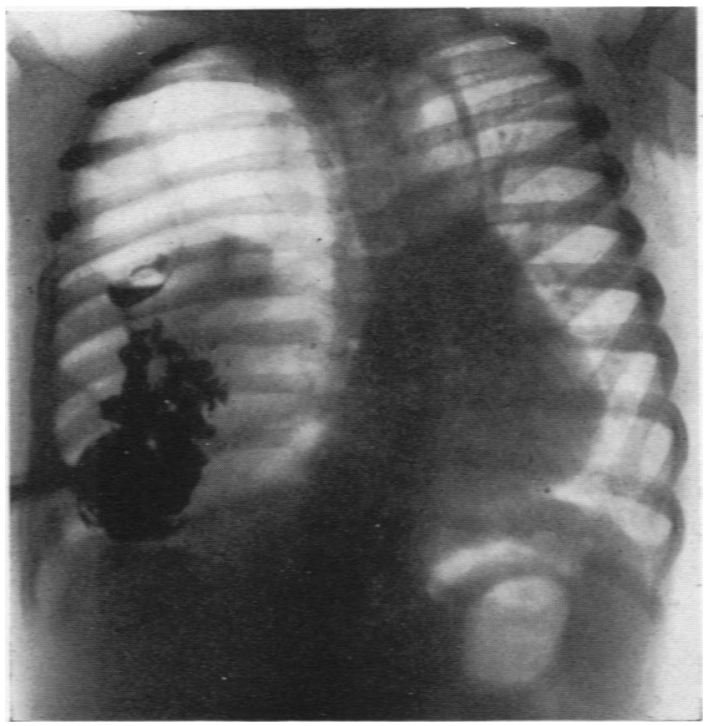

FK. 4.-Radiograph after the instillation of lipiodol into the empyema. Blobs of lipiodol represent either loculations or pulmonary alveoli. A narrow channel communicates with the pneumothorax above.

pleural cavity by means of a record syringe using gentle pressure, and a radiograph was taken. Lipiodol was shown to be entering the right pleural cavity and to be filling what appeared to be loculations. The curious appearance of blobs of lipiodol was difficult to interpret. Loculations was one suggestion, another was a filling up of pulmonary alveoli. Careful study of the film taken on March 18 after lipiodol had been instilled also demonstrated a narrow channel leading upwards from the lower blobs of lipiodol, which seemed to enter an

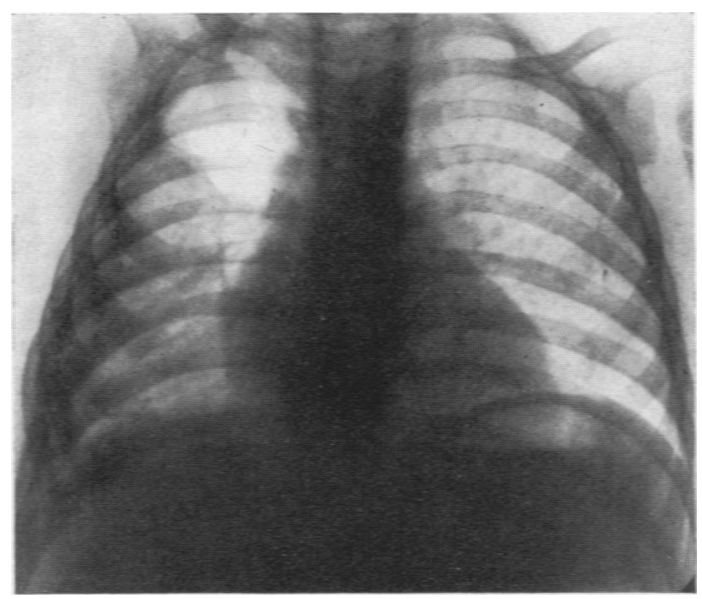

FKG. 5.-Radiograph after the release of air from the tension pneumothorax. The heart and mediastinum occupy normal positions. air-filled cavity occupying the right upper thorax. There was a small pool of lipiodol situated at the bottom of the cavity presenting a fluid level and a thin line of lipiodol creeping up the side of the cavity by surface tension. The upper half of the right side of the chest was completely translucent, strongly suggesting a pneumothorax. The translucent area extended across the mid-line to the left side of the chest. The whole of the mediastinum and the heart were markedly displaced towards the left side (Fig. 4).

At this stage very little pus was draining from the empyema tube and it was decided to establish free drainage by rib resection. This was performed on March 20. A de Pezzer catheter was stitched into the pleura. The skin was closed around the catheter which was led as before to an underwater drain. In spite of this there was still very little drainage from the pleura. Following the rib resection the child became pyrexial

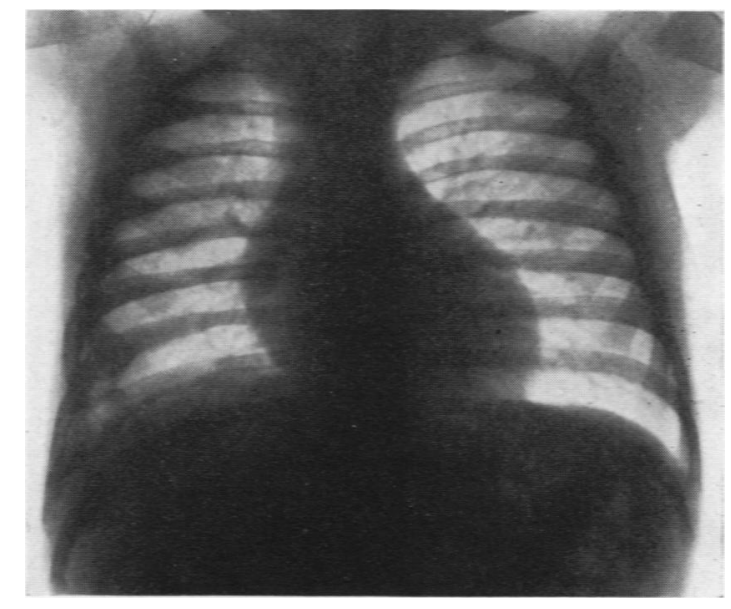

Fig. 6. - Radiograph showing complete re-expansion of the right lung.

and a second course of streptomycin was given from March 21 to 31 .

Her condition remained unsatisfactory. Although the temperature was normal, she was cyanosed and obviously distressed. It was realized at this point that radiographs taken on March 18 were very strongly suggestive of a tension pneumothorax. Figs. 2 and 3 are radiographs taken on March 7 and 14 respectively. They were taken before the instillation of lipiodol. The radiograph of March 18 (Fig. 4) was taken after the instillation of lipiodol. All demonstrated the presence of a tension pneumothorax. The radiograph of March 14 demonstrated an adhesion crossing the right side of the pneumothorax from the upper portion of the collapsed right lung to the chest wall (Fig. 3).

Accordingly, on March 21, we began the release of the tension on the right side of the upper chest. A cannula was inserted into the third intercostal space anteriorly and attached to an artificial pneumothorax apparatus 
based on the principle of Lillingston and Pearson. A pressure reading was taken at 8.30 p.m. The pressure excursion was very great. Air was released at intervals until the pressure was normal (see Table).

The tension pneumothorax seemed to re-accumulate air after each aspiration very slowly. The conclusion was that there was a bronchopleural fistula or a valvular communication from the empyema cavity to the upper pleura. No further drainage occurred from the empyema tube and this was removed on March 30. The sinus stopped discharging on about April 11.

Although there had been a history of only five days beginning with coryza, when the child was first seen in hospital an empyema thoracis had developed. The history suggested an upper respiratory infection going on to pneumonia followed rapidly by empyema, which was confirmed by paracentesis thoracis. The identification of Staphylococcus aureus completed the diagnosis. There was pyrexia during the first nine days, the morning and evening temperature reaching between 100 and $101^{\circ} \mathrm{F}$.

The progress of the case might be conveniently divided into three phases. First, from the time of diagnosis until simple daily aspiration of the chest was abandoned, February 25 to March 1 . The fluid was already purulent on admission and it was difficult to obtain adequate drainage by syringe aspiration, but it was possible to instill penicillin within the empyema cavity on three occasions.

Systemic chemotherapy extended into the second phase beginning when intercostal catheter drainage was instituted on March 1. At first, drainage under water was successful. Penicillin instillation was attempted, but failure to drain successfully necessitated frequent readjustments of the catheter. Lipiodol was run into the catheter and loculations of pus demonstrated and rib resection was undertaken. The patient was almost apyrexial during this period. There was an occasional temperature of $99:$ F. Following rib resection, the temperature rose but fever was controlled by giving streptomycin.

The third phase was from the time of recognition of the fundamental cause of the respiratory distress and cyanosis, i.e. the tension pneumothorax. This was released during the succeeding $\mathbf{1 0}$ days.

There is no doubt in retrospect that valuable time was lost in the second phase of the treatment (March 1 to 21). The intrapleural loculations interfered with drainage and prevented adequate penicillin instillations after the technique described by Fatti, Florey, Joules, Humphrey and Sakula (1946). Moreover, the presence of a tension pneumothorax was not recognized as early as it should have been.

The total time from intercostal drainage to the healing of the sinus was six weeks. Fatti et al. (1946) in 20 cases treated by a specially described technique of penicillin instillations through an intercostal drain, and compared with 14 controls obtained healing of the sinus three to six weeks after the beginning of drainage compared with an average of 11.6 weeks in the controls. The time from earliest pneumonic symptoms to healing of the sinus was seven weeks against 15 weeks in control cases untreated by pencillin instillations.

Once, however, adequate drainage was obtained by rib resection and loculations broken down and adequate steps had been taken to deal with the tension pneumothorax, recovery took place very quickly.

\section{Summary}

A case of tension pneumothorax and empyema

RANGE OF PRESSURE EXCURSIONS

\begin{tabular}{|c|c|c|c|c|c|c|c|}
\hline \multirow[t]{2}{*}{$\begin{array}{l}\text { Date and } \\
\text { Time }\end{array}$} & \multirow{2}{*}{$\begin{array}{c}\text { Amount of Air } \\
\text { Withdrawn } \\
\text { (ml.) }\end{array}$} & \multicolumn{2}{|c|}{ Range of Pressure in Pleura* } & \multicolumn{2}{|l|}{ Pulse } & \multicolumn{2}{|c|}{ Respirations } \\
\hline & & $\begin{array}{c}\text { Before } \\
\text { (cm. water) }\end{array}$ & After & Before & After & Before & After \\
\hline $\begin{array}{l}21.3 .52 \\
6.10 \text { p.m. }\end{array}$ & 325 & $-7 /-9$ & $-7 /-8$ & 130 & 150 & 40 & 50 \\
\hline 8.30 p.m. & 100 & $-9 /-2$ & $-8-5$ & 138 & 160 & 44 & 54 \\
\hline 9.30 p.m. & 100 & $-10 /-5$ & $-5 /-5$ & 130 & 164 & 44 & 54 \\
\hline 10.30 p.m. & 75 & $-7 /-5$ & $-5 /-5$ & 132 & 160 & 48 & 58 \\
\hline 11.30 p.m. & 125 & $-9 /-2$ & $-6 i-4$ & 142 & 150 & 48 & 54 \\
\hline $\begin{array}{l}22.3 .52 \\
10.30 \text { a.m. }\end{array}$ & 100 & $-10 /-4$ & $-4,-4$ & 150 & 134 & 58 & 54 \\
\hline 7.30 p.m. & 175 & $-4 i-6$ & $-5-5$ & 146 & 154 & 50 & 56 \\
\hline 23.3 .52 & & & & Approximately & 150 & Ap & 50 \\
\hline 24.3 .52 & & & & Approximately & 145 & Ap & $35-58$ \\
\hline 25.3 .52 & 175 & $-4 /-2$ & $-4-8$ & 140 & 148 & 32 & 48 \\
\hline 26.3 .52 & $\mathbf{0}$ & $-2 i-8$ & & 128 to & 140 & 26 to & 48 \\
\hline 30.3 .52 & 10 & $-1 /-4$ & $-1 /-5$ & 126 & 138 & 26 & 38 \\
\hline
\end{tabular}

* Normal intrapleural pressure is $-7 \mathrm{~cm}$. water in expiration, $-14 \mathrm{~cm}$. water in inspiration. 
following primary staphylococcal pneumonia has been described.

It is pointed out that in cases of staphylococcal empyema it should be borne in mind that lung abscess, tension cyst, or tension pneumothorax is a distinct possibility. This helps in making an early diagnosis.

Treatment of the tension pneumothorax is described, making use of the Lillingston and Pearson artificial pneumothorax apparatus.
REFERENCES

Blumenthal, S. and Neuhof, H. (1946). Amer. J. Dis. Child., 72, 691. Chickering, H. T. and Park, J. H. (1919). J. Amer. med. Ass., 72, 617.

Clemens, H. H. and Weens, H. S. (1942). J. Pediat., 20, 281.

Davis, W. S., Hyman, M.E. and Ruhstaller, F. D. (1947). J. Pediat., 30, 55 .

Dickie, A. E. (1938). Lancet, 1, 139.

Fatti, L., Florey, M. E., Joules, H., Humphrey, J. H. and Sakula, J. (i946). Lancet, 1, 295.

Gibson, M. O. J. and Belcher, J. R. (1951). Quart. J. Med., 20, 43. Guthrie, K. J. and Montgomery, G. L. (1947). Lancet, 2 i 52.

Hochberg. L. A. and Kramer, B. (1939). Amer. J. Dis. Child., 57. 1310.

Kanof, A., Kramer, B. and Carnes, M. (1939). J. Pediat., 14, 712. Ladd, W. E. and Swan, H. (1943). Ibid., 23, 297.

Macgregor, A. R. (1936). Archives of Disease in Childhood, 11, 195.

Riley, C. M. (1944). J. Pediat., 24, 577. 